\title{
Evidence for improved control of hypertension in Taiwan: 1993-2002
}

\author{
Ta-Chen Su ${ }^{a}$, Chyi-Huey Bai ${ }^{b}$, Hsing-Yi Chang ${ }^{c}$, San-Lin You ${ }^{d}$, \\ Kuo-Liong Chien ${ }^{\mathrm{a}, \mathrm{e}}$, Ming-Fong Chen ${ }^{\mathrm{a}}$, Hsin-Jen Chen ${ }^{f}$, Wen-Harn Pan ${ }^{\dagger}$, \\ Chin-Hsiao Tseng ${ }^{a}$, Shou-Hsia Cheng ${ }^{g}$, Baai-Shyun Hurngh, \\ Lee-Ching Hwang and Chien-Jen Chen ${ }^{i}$
}

\begin{abstract}
Objective This study reports the prevalence of hypertension, proportions of awareness, treatment, and control in the 2002 Taiwanese Survey on Hypertension, Hyperglycemia, and Hyperlipidemia (TwSHHH), and compared the changes of hypertension prevalence, awareness, treatment, and control in two recent nationwide surveys.
\end{abstract}

\begin{abstract}
Methods TwSHHH is the second nationwide survey designed to assess the prevalence, awareness, treatment, and control of hyperglycemia, hyperlipidemia, and hypertension. The TwSHHH survey applied a multistage, stratified, and random sampling during 2002 with a total of 7566 participants. Among them, 3088 male and 3391 female participants were 19 years old and over and were selected from households throughout Taiwan. The data of Nutrition and Health Survey in Taiwan (NAHSIT), the first nationwide survey to assess disease and nutrition status during 19931996, was also applied to compare changes of the prevalence, awareness, treatment, and control of hypertension between the two surveys.
\end{abstract}

Results Compared with the NAHSIT, the prevalence of hypertension on TwSHHH decreased significantly in female adults, between 1993-1996 and 2002. In both males and females of all age groups, the awareness, treatment, and control of hypertension significantly and substantially improved between NAHSIT and TwSHHH. These results also correlated in time with the implementation of National Health Insurance since 1995. The favorable

\section{Introduction}

The Nutrition and Health Survey in Taiwan (NAHSIT) was developed to assess disease and nutrition status during 1993-1996 [1], an interval of time that followed 20-30 years of rapid economic growth in Taiwan. Hypertension was a major focus of the survey due to the possibility that dietary and lifestyle changes associated with economic growth increased the incidence and prevalence of cardiovascular disease (CVD). Data from the NAHSIT indicated that the prevalence of hypertension is on the rise, especially in rural areas where access to medical care is less and therefore rates of awareness, treatment, control, and medication compliance are correspondingly lower. Prior to the institution of the National changes in education and availability of care may account for improved control of hypertension and, possibly, its prevention.

Conclusions There was a significant improvement of hypertension awareness, treatment, and control in the TwSHHH survey compared with the NAHSIT survey in Taiwan. J Hypertens 26:600-606 @ 2008 Wolters Kluwer Health | Lippincott Williams \& Wilkins.

Journal of Hypertension 2008, 26:600-606

Keywords: awareness, control, hypertension, prevalence, treatment

Abbreviations: BMI, body mass index; DBP, diastolic blood pressure; JNC-7, The Seventh Report of the Joint National Committee on Prevention, Detection, Evaluation and Treatment of High Blood Pressure; NAHSIT, Nutrition and Health Survey in Taiwan; PPS, probability proportional to size: SBP, systolic blood pressure; TwSHHH, Taiwan Survey on Hypertension, Hyperglycemia, and Hyperlipidemia

${ }^{a}$ Department of Internal Medicine, National Taiwan University Hospital and National Taiwan University College of Medicine, ${ }^{b}$ Central Laboratory, Shin Kong WHS Memorial Hospital, ' Division of Health Policy Research, National Health Research Institutes, ${ }^{d}$ Graduate Institute of Epidemiology, College of Public Health, National Taiwan University, ${ }^{e}$ Graduate Institute of Preventive Medicine, College of Public Health, National Taiwan University, Institute of Biomedical Sciences, Academia Sinica, ${ }^{9}$ Institute of Health Policy \& Management and Center for Health Policy Research, College of Public Health, National Taiwan University, hBureau of Health Promotion, Department of Health, 'Department of Family Medicine, McKay Memorial Hospital and ${ }^{\mathrm{j}}$ Genomics Research Center, Academia Sinica, Taipei, Taiwan

Correspondence to Chien-Jen Chen, MPH, ScD, Genomics Research Center, Academia Sinica, 128 Academia Road Section 2, Nankang, Taipei 11529, Taiwan Tel: +886 22737 7750; e-mail: cjchen@ntu.edu.tw

Received 12 March 2007 Revised 10 October 2007 Accepted 23 October 2007

Health Insurance (NHI) program, only $57 \%$ of Taiwanese were covered by medical insurance [2]. Then in 1995, when the NHI program was implemented, medical services became more accessible in Taiwan. As a result, $96 \%$ of citizens in Taiwan are covered under NHI and $90 \%$ of hospitals and clinics were NHIcontracted providers by 1996 [2,3].

The NAHSIT data were largely collected before the actual availability of NHI implementation. Consequently, to determine the effect of NHI on public health, the Taiwanese Survey on Hypertension, Hyperglycemia, and Hyperlipidemia ( TwSHHH) was conducted in 2002 and not only updated information about the prevalence of 
hypertension in Taiwan, but also allowed investigators to examine the influence of NHI on the prevalence of hypertension, disease control, and changes in medical behaviors in nationwide samples [4-6].

By accessing data from the TwSHHH, the goals of the current study were to establish the current prevalence, awareness, treatment, and control of hypertension in the general population of Taiwan and to compare changes in the prevalence, awareness, treatment, and control of hypertension before and after implementation of NHI since 1995.

\section{Methods}

\section{Study design}

Study subjects were randomly selected from the National Health Interview Survey (NHIS) of Taiwan (2001) conducted by the National Health Research Institutes and Bureau of Health Promotion, Taiwan [4-6]. The NHIS incorporated a multistaged stratified systematic sampling scheme. It first divided 359 townships or districts of Taiwan into seven strata according to geographic location and degree of urbanization. Townships or districts in each stratum were selected by probability proportional to their size (PPS). In each selected township/district, lins (the smallest administrative unit) were selected by PPS. Four households were selected randomly from each selected lin [5].

Ultimately, 6592 households from 1648 townships were included, yielding an estimated 23473 people of 15 years or older. Because use of this number of participants was not economically feasible for the TwSHHH, one-half of the townships from 21 counties and 54 villages, towns, cities, and districts were randomly selected. After crosschecking with data of the NHIS, the final recognized effective representative samples were 10292 . Among them were 9659 people of age 19 years or older representing 3296 households from 824 townships who were eligible for study. In actuality, a total of 6479 adults (3088 males and 3391 females) permitted blood pressure (BP) measurements and enrolled in this analysis. All study subjects were 19 years or older, previous interviewees of the NHIS conducted between August 2001 and January 2002, and provided informed consent for BP determination and venipuncture for fasting blood lipids, and sugar measurements. Residents in military housing communities, medical facilities, schools, job training centers, dormitories, prisons, or residents of rural areas and offshore islands were excluded from participating in the study. The major reason for not participating was declining the interview by 1775 individuals.

\section{Blood pressure measurement}

Two BP measurements were obtained by certified interviewers as recommended by the American Heart Association [7]. If the two BP measurements differed by more than $10 \mathrm{mmHg}$, a third measurement was made, and the two closest measurements were then averaged. BPs were standardized by having an interviewer trainee and teaching assistant take the $\mathrm{BP}$ of the same subject using a binaural stethoscope and simultaneously recording both findings. Trainees were considered qualified only when the difference between their findings and the trainer's findings were within $2 \mathrm{mmHg}$. The measurement was taken with a calibrated mercury sphygmomanometer and cuffs of the appropriate size, with the participant seated and at rest for 5-10 min, and with the participant's arm raised to the same height as the heart. The participant was further advised not to walk, run, or lift heavy objects within $30 \mathrm{~min}$ of BP measurement. Both NAHSIT and TwSHHH surveys used the similar procedure of BP measurements and questionnaires. The methods of obtaining the results of questionnaire by inquiring awareness, treatment, and control of hypertension between these two surveys were similar. Therefore, the results were considered comparable.

Hypertension was evaluated according to the seventh report of the Joint National Committee on Prevention, Detection, Evaluation, and Treatment of High BP (JNC-7) [8]. Hypertension was defined as an average systolic BP (SBP) of $140 \mathrm{mmHg}$ or higher or an average diastolic BP (DBP) of $90 \mathrm{mmHg}$ or higher or self-reported use of antihypertensive medications. Awareness of hypertension was defined as a self-report of a high BP measurement in the past. Treatment of hypertension was defined as use of a prescription medication to manage high $\mathrm{BP}$ at the time of the interview. Control of hypertension was defined as pharmacological treatment of hypertension associated with an average SBP of under $140 \mathrm{mmHg}$ and an average DBP of under $90 \mathrm{mmHg}$ during the TwSHHH survey.

\section{Data analysis}

In analyzing the data, $\mathrm{BP}$ was expressed as the mean \pm 1 SD of SBP and DBP. Sex and age-specific estimates of normotension ( $\mathrm{SBP} \leq 120 \mathrm{mmHg}$ and $\mathrm{DBP} \leq 80 \mathrm{mmHg}$ ), prehypertension (SBP $120-139 \mathrm{mmHg}$ or DBP $80-$ $89 \mathrm{mmHg}$ ), stage I hypertension (SBP $140-159 \mathrm{mmHg}$ or DBP $90-99 \mathrm{mmHg}$ ), and stage II hypertension (SBP $\geq 160 \mathrm{mmHg}$ or DBP $\geq 100 \mathrm{mmHg}$ ) according to JNC-7 are provided in Table 1 [8].

The prevalence of hypertension was compared between two surveys according to different strategies. The mean and standard error (SE) of the prevalence was estimated using SUDAAN 9.0 to account for the complex sampling scheme. Two-sample $t$-test was used after obtaining the $\mathrm{SE}$ of each estimate. Bonferroni adjustment was used to account for multiple comparisons. In this study, three age groups were compared, their significance level $(0.05)$ was divided by three. Only with a $P$ value smaller than $0.05 / 3$ was statistical significance reached. 
Table 1 Distribution of blood pressure classes in Taiwanese in the Taiwanese Survey on Hypertension, Hyperglycemia, and Hyperlipidemia according to the diagnostic criteria of the Seventh Report of the Joint National Committee on Prevention, Detection, Evaluation and Treatment of High Blood Pressure

\begin{tabular}{|c|c|c|c|c|c|}
\hline & \multicolumn{3}{|c|}{ Normotension or controlled hypertension } & \multicolumn{2}{|c|}{ Hypertension } \\
\hline & & Normal (\%) & Prehypertension (\%) & Stage1 (\%) & Stage $2(\%)$ \\
\hline $\mathrm{SBP} \mathrm{mmHg}$ & & $<120$ & $120-139$ & $140-159$ & $>160$ \\
\hline DBP $(\mathrm{mmHg})$ & & $<80$ & $80-89$ & $90-99$ & $>100$ \\
\hline \multicolumn{6}{|l|}{ Combined age } \\
\hline & $19-44$ & 71.53 & 22.12 & 4.69 & 1.66 \\
\hline & $45-64$ & 39.58 & 36.38 & 18.07 & 5.98 \\
\hline & $\geq 65$ & 20.90 & 42.23 & 25.82 & 11.05 \\
\hline & $\geq 19$ & 53.68 & 29.62 & 12.24 & 4.46 \\
\hline \multicolumn{6}{|l|}{ Male } \\
\hline & $19-44$ & 57.88 & 31.68 & 7.67 & 2.77 \\
\hline & $45-64$ & 34.23 & 38.04 & 19.90 & 7.84 \\
\hline & $\geq 65$ & 23.17 & 40.29 & 27.77 & 8.77 \\
\hline & $\geq 19$ & 44.62 & 34.88 & 15.06 & 5.44 \\
\hline \multicolumn{6}{|l|}{ Female } \\
\hline & $19-44$ & 84.08 & 13.32 & 1.96 & 0.64 \\
\hline & $45-64$ & 44.21 & 34.94 & 16.49 & 4.37 \\
\hline & $\geq 65$ & 18.39 & 44.37 & 23.68 & 13.56 \\
\hline & $\geq 19$ & 61.93 & 24.83 & 9.67 & 3.57 \\
\hline
\end{tabular}

The prevalence, awareness, treatment, and control of hypertension amongst the different age groups in the NAHSIT and TwSHHH were compared. A $t$-test was used to make comparisons between these groups. A $P$ value of less than 0.05 was considered statistically significant.

\section{Results}

Systolic blood pressure, diastolic blood pressure, pulse pressure, and hypertension prevalence in relation to age and sex in TwSHHH

Figure 1 shows the average values of $\mathrm{BP}$ components and prevalence of hypertension by age and sex. Overall, increases in average SBP and DBP were found to begin at ages 30-39 years. While the increase in SBP continued up to age 80 years, DBP peaked at ages $50-59$ years and decreased thereafter. In men, SBP peaked at ages 70 79 years while DBP peaked at ages 50-59 years, and decreased thereafter with age. In women, DBP peaked between 60 and 69 years, whereas SBP continued to increase beyond age 80 years. Pulse pressure increased with age for both men and women after age 30-39 years. The sex differences are evident with higher BP in males before age 60 years in SBP and before age 70 years in DBP. Compared with women, however, men had higher pulse pressure before age 40 years and crossover after age 50 years and before age 70 years, and there was no difference at ages $40-49$ years and after age 70 years. The hypertension prevalence increased with age and men had a higher prevalence before age 60 years.

\section{Hypertension and prehypertension prevalence in relation to age and gender in TwSHHH}

The sex and age-specific prevalence of prehypertension, stage I hypertension (SBP $140-159 \mathrm{mmHg}$ or DBP $90-$ $99 \mathrm{mmHg}$ ) and stage II hypertension (SBP $\geq 160 \mathrm{mmHg}$ or DBP $\geq 100 \mathrm{mmHg}$ ) were diagnosed according to the criteria of JNC-7. The prevalence of prehypertension and hypertension increased with age in both sexes, but the rapid increase of stage 2 hypertension in women after age 65 years was evident. After 45 years of age, prehypertension and hypertension increased in both men and women significantly. In general, about $35 \%$ of men and $25 \%$ of women had BP at the range of prehypertension, and about $21 \%$ of men and $13 \%$ of women with BP above $140 / 90 \mathrm{mmHg}$ even with antihypertensive medication in adults in Taiwan (Table 1).

\section{Hypertension prevalence and comparisons between two surveys according to different strategies}

For the comparison between two surveys, we used the US 2000 population as the reference population. Table 2 showed the prevalence of hypertension in the Taiwanese populations, comparing the hypertension prevalence between NAHSIT (1993-1996) and TwSHHH (2002) according to different classifications. In general, TwSHHH had lower hypertension prevalence in comparison with NAHSIT. Further analysis showed that TwSHHH had lower hypertension prevalence at ages 45-64 years. To our surprise, the major difference came from women but not men in all age groups. In addition, the hypertension prevalence in a male age group of 65 years or above was even higher in TwSHHH. Comparing with those in NAHSIT, age-adjusted hypertension prevalence decreased in all age groups of women in TwSHHH, yet there were no such significant findings in any age group of males. While comparing with NAHSIT, the hypertension prevalences were found to be significantly lower in the groups of overweight (BMI $24-26.9 \mathrm{~kg} / \mathrm{m}^{2}$ ) and obesity (BMI at and above $27 \mathrm{~kg} / \mathrm{m}^{2}$ ) in the 2002 TwSHHH. 


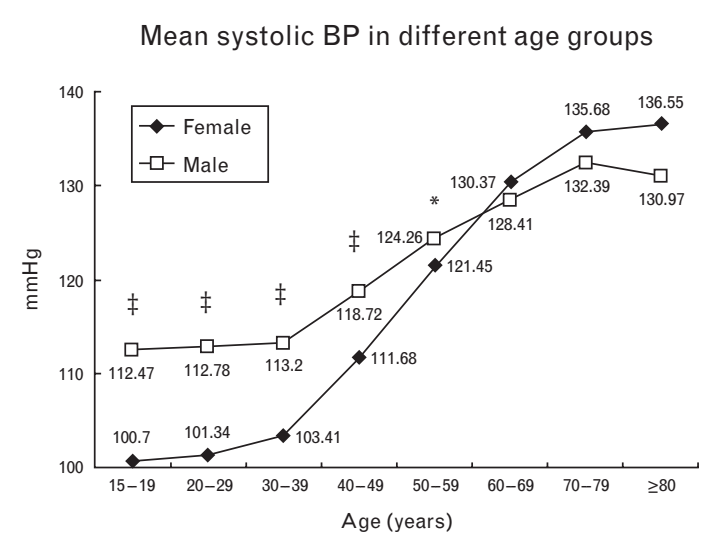

Mean pulse pressure in different age groups

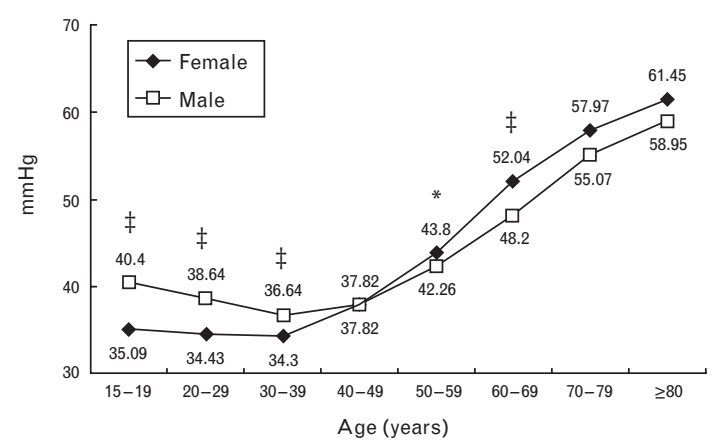

Mean diastolic BP in different age groups

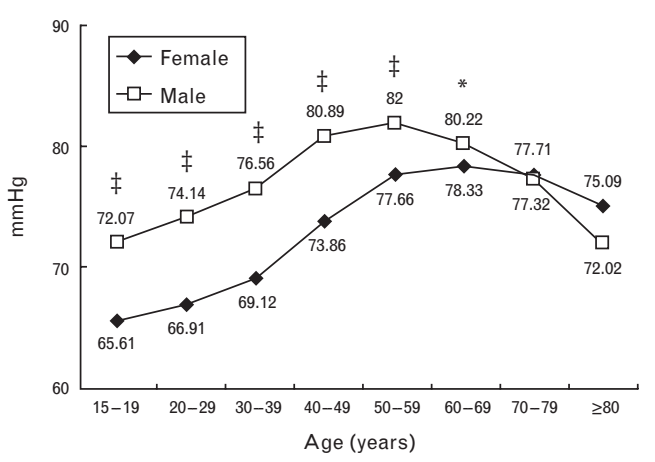

Prevalence of hypertension in different age groups

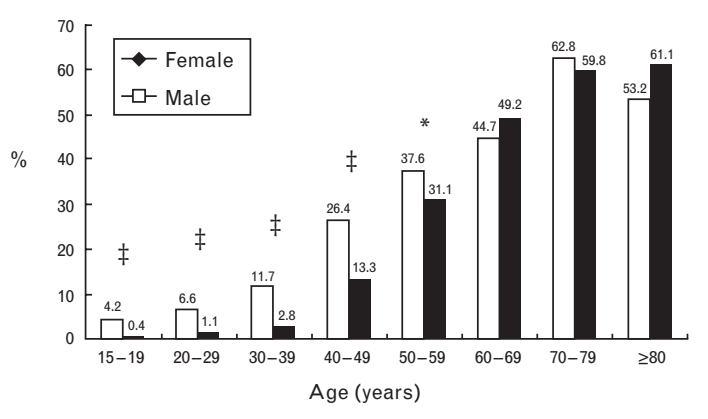

Systolic blood pressure (BP), diastolic BP, pulse pressure, and hypertension prevalence in relation to age and gender in $\mathrm{TwSHHH} .{ }^{\ddagger} P$-value $<0.005$, $* P$-value $<0.05$.

The prevalence of hypertension awareness, treatment, and control between two surveys

Table 3 shows a comparison of the characteristics of hypertension between the two surveys. Specifically, hypertension awareness, treatment, and control are listed. In men and women of all age groups, all three measures of hypertension significantly and substantially improved between NAHSIT and TwSHHH. These results also correlate in time with the NHI-related increase in coverage and utilization by those with little or no previous access to the medical care system.

\section{Discussion}

The most significant finding of this study was the decrease in hypertension prevalence and the improvement of hypertension awareness, treatment, and control during two nationwide surveys, indicating some favorable changes regarding the healthcare system may have occurred during this period, including health policies, the coverage of medical care system, and readily available medical services, of which the implementation of the NHI was the most important in Taiwan. In terms of better control of hypertension, this survey provides evidence of proactive healthcare policy that may positively impact on hypertension prevalence and well controlled hypertension. This study is a rare observation and comparison of a nationwide natural experiment of health policy on the health awareness and treatment of disease. This is the first survey to demonstrate the influence of a healthcare policy on a major disease in a nationwide sampling in the ethnic Chinese population. Such results can serve as a reference for healthcare policymakers.

Although the duration of the NAHSIT survey overlapped the timing of the initiation of NHI implementation, over half of the NAHSIT survey had been completed before March 1995. The health effects of the NHI implementation could not have happened immediately and will attenuate the higher prevalence of well controlled hypertension in TwSHHH. Thus, the NHI 
Table 2 Prevalence of hypertension in the Taiwanese populations, comparison between Nutrition and Health Survey in Taiwan (1993-1996) and Taiwanese Survey on Hypertension, Hyperglycemia, and Hyperlipidemia (2002)

\begin{tabular}{|c|c|c|c|c|}
\hline \multirow[b]{3}{*}{ Characteristics } & \multicolumn{4}{|c|}{ Hypertension prevalence, $\%$ (SE) } \\
\hline & \multicolumn{2}{|c|}{ NAHSIT } & \multicolumn{2}{|c|}{ TwSHHH } \\
\hline & Unadjusted & Age-adjusted $^{\mathrm{a}}$ & Unadjusted & Age-adjusted $^{a}$ \\
\hline Overall & $23.7(1.7)$ & $26.8(1.6)$ & $23.7(0.7)$ & $23.5(0.7)^{\mathrm{b}}$ \\
\hline \multicolumn{5}{|l|}{ Age, years } \\
\hline $19-44$ & $12.4(2.1)$ & $11.6(2.0)$ & $8.9(0.7)$ & $8.0(0.6)$ \\
\hline $45-64$ & $40.0(1.8)$ & $39.0(2.0)$ & $33.8(1.3)^{\mathrm{c}}$ & $34.1(1.3)^{b}$ \\
\hline$\geq 65$ & $56.2(2.5)$ & $57.7(3.6)$ & $57.1(1.9)$ & $58.1(1.9)$ \\
\hline \multicolumn{5}{|l|}{ Age by sex } \\
\hline Male & $26.4(2.3)$ & $28.3(2.2)$ & $28.0(1.0)$ & $27.1(0.9)$ \\
\hline $19-44$ & 16.6 & $15.9(3.0)$ & $14.0(1.0)$ & $12.9(1.0)$ \\
\hline $45-64$ & $41.3(2.7)$ & $40.9(2.8)$ & $37.2(1.7)$ & $37.6(1.7)$ \\
\hline$\geq 65$ & $50.0(6.0)$ & $49.1(7.0)$ & $56.8(2.5)$ & $57.9(2.5)^{\mathrm{b}}$ \\
\hline Female & $20.9(1.9)$ & $25.3(1.6)$ & $19.9(0.7)$ & $20.2(0.7)^{\mathrm{b}}$ \\
\hline $19-44$ & $8.1(1.8)$ & $7.2(1.6)$ & $4.3(0.5)^{\mathrm{c}}$ & $3.7(0.5)^{\mathrm{c}}$ \\
\hline $45-64$ & $37.7(2.3)$ & $37.1(2.5)$ & $30.8(1.4)^{\mathrm{c}}$ & $31.1(1.4)^{b}$ \\
\hline$\geq 65$ & 64.0 (3.3) & 67.2 (3.3) & $57.5(2.6)$ & $58.4(2.6)^{b}$ \\
\hline \multicolumn{5}{|l|}{$\mathrm{BMI}, \mathrm{kg} / \mathrm{m}^{2}$} \\
\hline$<24$ & $15.1(1.4)$ & $19.9(1.7)$ & $14.5(0.7)$ & $18.2(0.7)$ \\
\hline $24-27$ & $32.3(2.2)$ & $32.9(1.9)$ & $28.9(1.2)$ & $27.1(1.1)^{\mathrm{b}}$ \\
\hline$\geq 27$ & $57.8(4.7)$ & $49.4(4.7)$ & $37.8(1.6)^{\mathrm{c}}$ & $36.2(1.6)^{b}$ \\
\hline
\end{tabular}

NAHSIT, Nutrition and Health Survey in Taiwan; TwSHHH, Taiwanese Survey on Hypertension, Hyperglycemia, and Hyperlipidemia. ${ }^{a}$ The 2000 US population was used as the standard population. ${ }^{b}$ Significantly different NAHSIT for the adjusted prevalence, using Bonferroni adjustment for multiple comparisons. ${ }^{\mathrm{c}}$ Significantly different NAHSIT for the unadjusted prevalence, using Bonferroni adjustment for multiple comparisons.

implementation of hypertension awareness, treatment, and control on the NAHSIT survey might be not significant enough to alter our findings. It has been well documented that BP control leads to a reduction in the risk of CVD [8]. A meta-analysis of 61 cohort studies, involving 1000000 participants, demonstrated that small differences in BP can account for large differences in CVD outcomes [9]. For example, lowering SBP by $10 \mathrm{mmHg}$ or DBP by $5 \mathrm{mmHg}$ reduces the risk of stroke by about $35 \%$ and that of ischemic heart disease events by about $25 \%$ at age 65 years [10,11]. Despite this, demonstration of the relationship in specific populations is rare because of difficulties conducting the necessary large epidemiological surveys. Fortunately, a recent event facilitated such a study in Taiwan. In 1995, NHI was implemented, ushering in changes in many aspects of healthcare [2,3].

Before the NHI was introduced in 1995, uninsured people were those retired, elderly, disabled, women, students, and children. The uninsured were deterred from seeking necessary medical services, and this created unequal access to healthcare between socio-economic classes. After implementation of NHI in March 1995, these populations could access healthcare system without economic barriers [2]. Further supporting evidence comes from the changing mortality rate attributed to stroke, from $64.77 / 100000$ in 1994 to $53.46 / 100000$ in 2002 , and heart disease, from 56.93/100 000 in 1994 to $50.93 / 100000$ in 2002 [12]. These trends of a decreasing

Table 3 Trends in hypertension awareness, treatment, and control in the adult population during two nationwide surveys in Taiwan

\begin{tabular}{|c|c|c|c|c|c|c|}
\hline & \multicolumn{2}{|c|}{ Awareness, \% } & \multicolumn{2}{|c|}{ Treatment, \% } & \multicolumn{2}{|c|}{ Control, \% } \\
\hline & NAHSIT & TwSHHH & NAHSIT & TwSHHH & NAHSIT & TwSHHH \\
\hline \multicolumn{7}{|l|}{ Male } \\
\hline $19-44$ & 6.8 & $29.6^{\ddagger}$ & 0.9 & $15.5^{\ddagger}$ & 0.1 & $8.3^{\ddagger}$ \\
\hline $45-64$ & 35.0 & $56.8^{\ddagger}$ & 23.1 & $43.5^{\ddagger}$ & 2.9 & $18.7^{\ddagger}$ \\
\hline$\geq 65$ & 44.9 & $75.0^{\ddagger}$ & 31.9 & $67.8^{\ddagger}$ & 8.2 & $33.7^{\ddagger}$ \\
\hline$\geq 19$ & 22.5 & $55.8^{\ddagger}$ & 13.4 & $44.3^{\ddagger}$ & 2.4 & $21.0^{\ddagger}$ \\
\hline$\geq 45$ & 38.7 & $64.9^{\ddagger}$ & 26.4 & $54.3^{\ddagger}$ & 4.9 & $25.4^{\ddagger}$ \\
\hline \multicolumn{7}{|l|}{ Female } \\
\hline $19-44$ & 13.1 & $54.5^{\ddagger}$ & 3.2 & $28.8^{\ddagger}$ & 0.1 & $19.7^{\ddagger}$ \\
\hline $45-64$ & 45.7 & $72.3^{\ddagger}$ & 33.1 & $59.2^{\ddagger}$ & 7.8 & $27.1^{\ddagger}$ \\
\hline$\geq 65$ & 52.0 & $80.5^{\ddagger}$ & 40.0 & $68.0^{\ddagger}$ & 5.9 & $32.8^{\ddagger}$ \\
\hline$\geq 19$ & 39.3 & $73.6^{\ddagger}$ & 27.6 & $59.4^{\ddagger}$ & 5.1 & $28.5^{\ddagger}$ \\
\hline$\geq 45$ & 48.7 & $75.8^{\ddagger}$ & 36.3 & $63.0^{\ddagger}$ & 6.9 & $29.5^{\ddagger}$ \\
\hline
\end{tabular}

NAHSIT, Nutrition and Health Survey in Taiwan; TwSHHH, Taiwanese Survey on Hypertension, Hyperglycemia, and Hyperlipidemia. ${ }^{\ddagger} P<0.001$, for differences in hypertension awareness, treatment, and control between the NAHSIT and TwSHHH. 
mortality rate from CVD may be partly attributed to the decrease in hypertension prevalence and an increase in well controlled hypertension, as similar evidence from the United States demonstrated [13].

One important finding is the significant lower prevalence of awareness, treatment, and control in subjects aged 1944 years compared with middle or old age people, which may be because of greater concerns over health status among older people [13]. In addition, the significant lower prevalence of hypertension in overweight and obese populations in a later survey also indicated the greater concerns in this high-risk population. This indicates the urgent need for implementation of hypertension education for the 19-44 years old age group of patients. Some of the different effects on hypertension prevalence were proposed. The accessibility and availability of public health services also widely improved after NHI implementation. The prevalence decrease may be due partly to the initiation of nationwide adult health examination programs in NHI for those aged 40 years and above since 1997 , which provides preventive and proactive surveillance and health education for middle-aged and old-aged populations. Over the years, more clinics have been established in non-metropolitan areas. During these periods, the trends for changes in medical care institutions were that hospitals became larger in scale, and clinics spread out in numbers [14]. This trend should be beneficial to the upgrading of the national medical care standards. All of these changes provided Taiwanese with more opportunity to access public health resources and medical care. Thus, we may reasonably propose the implementation of NHI as the major reason for decreased hypertension prevalence and increased hypertension treatment and control.

One cohort survey of 1021 randomly selected adults found that after NHI was established those who were previously uninsured increased their use of outpatient visits to a comparable level to those with insurance [3]. These findings add support to the notion that increased accessibility and availability of medical services for the Taiwanese occurred after NHI implementation. As such, the control of hypertension and the prevention of CVD has become a feasible and important way to reduce morbidity and mortality. The prevalence of hypertension decreased significantly in women but not in men as found in this study also indicated the gender gap should be given more attention. Like the studies in Canada, the United States, and England, men are less aware of having hypertension and a lower rate receive antihypertensive treatment than women [15-17]. Thus, more studies are needed to investigate this gender difference.

Our results clearly demonstrate that healthcare policy can dramatically affect disease prevalence. In addition, the greatest improvement in both treatment and control of hypertension was achieved in the elderly, the age group most prone to CVD. Our study further showed that despite the NHI implementation, the high age-adjusted prevalence of hypertension persists among both men (27.1\%) and women (20.2\%). Although not quite comparable to the data derived from surveys of individuals 35 years or over, it has been reported that the prevalence of hypertension is $28 \%$ in North America, $44 \%$ in Europe [18], and 27.2\% in China [19]. The age-adjusted prevalence of hypertension for those aged 18 years or older in the US was $30.7 \%$ for men and $28.2 \%$ for women in $2003-$ 2004 [13]. In addition, in the current study it was shown that the prevalence of hypertension among middle-aged and elderly individuals ( $\geq 45$ years of age) remained high and the prevalence of receiving treatment and control of hypertension were still relatively low. Thus, hypertension remains a significant problem, especially in those middle-aged and elderly Taiwanese, who are amenable to proactive public health innovations.

The decline in hypertension prevalence in the US between 1960 and 1988 was similarly attributed to increases in hypertension awareness, treatment, and control [20]. Unfortunately, this decline has ceased in the US since 1988 [16]. Although Hajjar and Kotchen [16] attribute this finding and its attendant low hypertension control rates in part to a lack of awareness or treatment, they note that hypertension was controlled in only $53 \%$ of those who reported taking antihypertensive medication. Similarly, an analysis of the Framingham Study showed that hypertension was controlled in only $33.7 \%$ of those treated with medication [21]. Hajjar and Kotchen [16] further suggest that differences in health insurance coverage might explain the observed sex, race, and ethnic differences in awareness and control of hypertension. Despite the fact that the elderly (aged $\geq 65$ years) are covered by government-sponsored health insurance (i.e., Medicare) in the US, their study as well as other studies [22] found that hypertension control rates were paradoxically lowest in this group. Thus, from the viewpoint of cost-effectiveness for CVD prevention and control, implementation of NHI may be one of the choices.

The study limitations were as follows: the potential for misclassification in using NAHSIT 1993-1996; caveats of self report data; handling of caffeine, alcohol or tobacco use in measuring BP; comparability of estimates using 4-year data (NAHSIT, 1993-1996) versus 1-year data (TwSHHH, 2002); stability of variance estimates using 4 -year data versus 1-year data. The major difference of the two surveys was the time to complete the survey, NAHSIT 4 years and TwSHHH 1 year, which may associate with some variability in obtaining data from different research assistants and study nurses even though we had set up a standard protocol for conducting the questionnaire. 


\section{Acknowledgements}

We thank all public health professionals in the Health Centers of the representative villages in Taiwan for joining the Taiwanese Survey on Hypertension, Hyperglycemia, and Hyperlipidemia (TwSHHH) in 2002.

This study is based on the data from Taiwanese Survey on Hypertension, Hyperglycemia, and Hyperlipidemia (TwSHHH), provided by the Bureau of Health Promotion, Department of Health, R.O.C. (Taiwan). The descriptions or conclusions herein do not represent the viewpoint of the Bureau.

This study was supported by a grant from the Bureau of Health Promotion, Department of Health, Executive Yuan, Taiwan, R.O.C. (DOH-2002).

There are no conflicts of intrest.

\section{References}

1 Pan WH, Chang HY, Yeh WT, Hsiao SY, Hung YT. Prevalence, awareness, treatment and control of hypertension in Taiwan: results of Nutrition and Health Survey in Taiwan (NAHSIT) 1993-1996. J Hum Hypertens 2001; 15:793-798.

2 Rachel Lu JF, Hsiao WC. Does universal Health insurance make healthcare unaffordable? Lessons from Taiwan. Health Affairs 2003; 22:77-88.

3 Cheng $\mathrm{SH}$, Chiang TL. The effect of universal health insurance on healthcare utilization in Taiwan. Results from a natural experiment. JAMA 1997; 278:89-93.

4 Shih YT, Hung YT, Chang HY, Liu JP, Lin HS, Chang MC, et al. The design, contents, operation and the characteristics of the respondents of the 2001 National Health Interview Survey in Taiwan. Taiwan J Public Health 2004; 22:419-430; (In Chinese).

5 National Health Research Institutes. National Health Interview Survey (NHIS) in 2001, Taiwan. Bureau of Health Promotion and National Health Research Institutes, 2002. http://nhis.nhri.org.tw. [Accessed 2 November 2007].

6 Wen CP, Levy DT, Cheng TY, Hsu CC, Tsai SP. Smoking behaviour in Taiwan, 2001. Tob Control 2005; 14 (Suppl 1):i51-i55.

7 Perloff D, Grim C, Flack J, Frohlich ED, Hill M, McDonald M, Morgenstern BZ. Human blood pressure determination by sphygmomanometry. Circulation 1993; 88 (5 Pt 1):2460-2470.

8 Chobanian AV, Bakris GL, Black HR, Cushman WC, Green LA, Izzo JL Jr, et al. National Heart, Lung, and Blood Institute Joint National Committee on Prevention, Detection, Evaluation, and Treatment of High Blood Pressure. National High Blood Pressure Education Program Coordinating Committee. The Seventh Report of the Joint National Committee on Prevention, Detection, Evaluation and Treatment of High Blood Pressure: The JNC 7 Report. JAMA 2003; 289:2560-2571.

9 Lewington S, Clarke R, Qizilbash N, Peto R, Collins R, Prospective Studies Collaboration. Age-specific relevance of usual blood pressure to vascular mortality: a meta-analysis of individual data for one million adults in 61 prospective studies. Lancet 2002; 360:1903-1913.

10 Asia Pacific Cohort Studies Collaboration. Blood pressure and cardiovascular disease in the Asia Pacific region. J Hypertens 2003; 21:707-716.

11 MacMahon S, Peto R, Cutler J, Collins R, Sorlie P, Neaton J, et al. Blood pressure, stroke and coronary heart disease: part 1, prolonged differences in blood pressure: prospective observational studies corrected for the regression dilution bias. Lancet 1990; 335:765-774.

12 Vital statistics, Department of Health, Executive Yuan, Taiwan, R.O.C., 2006. http://www.doh.gov.tw/EN2006/DM/

DM2.aspx?now fod list no $=9085 \&$ class no $=390 \&$ level no $=1$ [Accessed 12 November 2007].

13 Ong KL, Cheung BM, Man YB, Lau CP, Lam KS. Prevalence, awareness, treatment, and control of hypertension among United States adults $1999-$ 2004. Hypertension 2007; 49:69-75.

14 Essential Statistical data of National Health Insurance, Department of Health, Taiwan, R.O.C., 2006. http://www.doh.gov.tw/EN2006/DM/ DM2.aspx?now_fod_list_no $=9085 \&$ class_no $=390 \& l e v e l \_n o=3$ [Accessed 12 November 2007].
15 Onysko J, Maxwell C, Eliasziw M, Zhang JX, Johansen H, Campbell NR. Canadian Hypertension Education Program. Large increases in hypertension diagnosis and treatment in Canada after a healthcare professional education program. Hypertension 2006; 48:853-860.

16 Hajjar I, Kotchen TA. Trends in prevalence, awareness, treatment, and control of hypertension in the United States, 1988-2000. JAMA 2003; 290:199-206.

17 Primatesta $\mathrm{P}$, Poulter NR. Improvement in hypertension management in England: results from the Health Survey for England 2003. J Hypertens 2006; 24:1193-1200.

18 Wolf-Maier K, Cooper RS, Banegas JR, Giampaoli S, Hense HW, Joffres M, et al. Hypertension prevalence and blood pressure levels in 6 European countries, Canada, and the United States. JAMA 2003; 289:2363-2369.

19 Gu D, Reynolds K, Wu X, Chen J, Duan X, Muntner P, et al., InterASIA Collaborative Group. The International Collaborative Study of Cardiovascular Disease in ASIA. Prevalence, awareness, treatment, and control of hypertension in China. Hypertension 2002; 40:920-927.

20 Burt VL, Cutler JA, Higgins M, Horan MJ, Labarthe D, Whelton P, et al. Trends in the prevalence, awareness, treatment, and control of hypertension in the adult US population. Data from the health examination surveys, 1960 to 1991. Hypertension 1995; 26:60-69.

21 Lloyd-Jones DM, Evans JC, Larson MG, Levy D. Treatment and control of hypertension in the community: a prospective analysis. Hypertension 2002 40:640-646

22 Hyman DJ, Pavlik VN. Characteristics of patients with uncontrolled hypertension in the United States. N Engl J Med 2001; 345:479-486. 